\title{
The Use of Metabolism Versus Partition Coefficient in Predicting Human Intestinal Permeability Classification
}

\author{
Nasir Idkaidek* \\ College of Pharmacy, Petra University, Jordan
}

Submission: March 30, 2017; Published: May 02, 2017

*Corresponding author: Nasir Idkaidek, Department of Pharmacy, Petra University, Jordan, Email: nidkaidek@uop.edu.jo

\begin{abstract}
Aim: The aim of this review commentary is to compare measured permeability class versus predicted one using metabolism and partition coefficient values for WHO essential drugs list.

Methods: Predicted permeability classes were based on partition coefficient or metabolism. Comparisons were then done against measured permeability classes.

Results and discussion: Predictions were close to measured permeability classes for many drugs using all prediction methods using metoprolol coefficients as reference marker and high extraction ratio for high permeability. However, some false negative and false positive predictions were observed. This can lead to wrong permeability classification and hence wrong biopharmaceutics classification (BCS). The best method with least false predictions in the studied compounds was using more than $90 \%$ metabolism as indicator of high permeability, Hence it is suggested to be used for better permeability classification prediction.
\end{abstract}

Keywords: Metabolism; Permeability; Partition coefficient; BCS; BDDCS

\section{Background}

Biopharmaceutics classification system (BCS) has classified drugs into four classes based on permeability and solubility [1]. BCS has become a corner stone in waiver of bioequivalence studies of generic drug products. Solubility class is usually easy to figure out, but permeability is not. Over the last 10 years, some trials were done to help predict human permeability class using drug partition coefficient and drug metabolism. However, limitations were observed due to existence of false positive and false negative predictions. Moreover, some differences were observed between BCS and the proposed biopharmaceutics drug disposition classification system (BDDCS) that has classified drugs into four classes based on metabolism and solubility [2-4]. US FDA has issued guideline for waiver of classes I and III drugs with support of permeability and solubility classes [5]. With this dilemma in mind, one needs a good way to predict correct permeability class and hence correct BCS class I or III.

\section{Objectives}

The aim of this review commentary is to compare measured permeability class versus predicted one using metabolism, diffusion and partition coefficient values for WHO essential drugs list.

\section{Methods}

Predicted permeability classes for 29 compounds from WHO essential drugs list were based on partition coefficient or metabolism. Metoprolol partition coefficient was used as reference marker compound. Hence, all values above metoprolol are classified as high permeability classes. However, high extraction ratio drugs with more than $70 \%$ metabolism are classified as high permeability classes. Then, drugs with more than $90 \%$ metabolism are classified as high permeability classes. Comparisons were then done against measured permeability classes to investigate the best method of prediction. Percent prediction error was calculated using the equation:

$\%$ Error $=$ (number of wrong predictions / total number) $\mathrm{X} 100$

\section{Results and Discussion}

Predictions were close to measured permeability classes for many drugs using all prediction methods using metoprolol as reference marker and high extraction ratio for high permeability. However, some false negative and false positive predictions were observed as shown in (Table 1). 8 drugs showed false predictions with $27.5 \%$ prediction error when using partition coefficient alone. This can lead to wrong permeability classification and 


\section{Novel Approaches in Drug Designing \& Development}

hence wrong biopharmaceutics classification (BCS). On the other hand, only 2 drugs showed false predictions with 6.9 $\%$ prediction error when using more than $70 \%$ metabolism

predictions with $3.5 \%$ prediction error when using more than $90 \%$ metabolism as cutoff of high permeability. The best method as cutoff of high permeability, while only 1 drug showed false more than $90 \%$ metabolism as indicator of high permeability.

Table 1: Measured versus predicted permeability of for the studied WHO essential drugs list.

\begin{tabular}{|c|c|c|c|c|c|c|}
\hline Name* & $\log P$ & $\begin{array}{l}\text { Peff-Pred } \\
\text { Metoprolol } \\
\text { Reference }\end{array}$ & $\begin{array}{l}\text { Peff-Pred: } \\
\mathbf{7 0 \%} \text { based } \\
\text { Metabolism }\end{array}$ & $\begin{array}{l}\text { Peff-Pred: } \\
\text { 90\% based } \\
\text { Metabolism }\end{array}$ & $\begin{array}{c}\text { Peff } X 1^{-4} \mathrm{~cm} / \\
\text { sec }\end{array}$ & $\begin{array}{l}\text { Peff-Class } \\
\text { Measured }\end{array}$ \\
\hline methyldopa & 0.39 & $\mathrm{~L}$ & $\mathrm{~L}$ & $\mathrm{~L}$ & 0.1 & $\mathrm{~L}$ \\
\hline amoxicillin & -0.58 & $\mathrm{~L}$ & $\mathrm{~L}$ & $\mathrm{~L}$ & 0.3 & $\mathrm{~L}$ \\
\hline antipyrine & 1.01 & $\mathrm{~L}$ & $\mathrm{H}$ & $\mathrm{H}$ & 5.6 & $\mathrm{H}$ \\
\hline Atenolol & 0.5 & $\mathrm{~L}$ & $\mathrm{~L}$ & $\mathrm{~L}$ & 0.2 & $\mathrm{~L}$ \\
\hline carbamazepine & 2.93 & $\mathrm{H}$ & $\mathrm{H}$ & $\mathrm{H}$ & 4.3 & $\mathrm{H}$ \\
\hline cephalexin & -0.67 & $\mathrm{~L}$ & $\mathrm{~L}$ & $\mathrm{~L}$ & 1.56 & $\mathrm{H}$ \\
\hline cimetidine & 0.79 & $\mathrm{~L}$ & $\mathrm{~L}$ & $\mathrm{~L}$ & 0.26 & $\mathrm{~L}$ \\
\hline creatinine & -0.63 & $\mathrm{~L}$ & $\mathrm{~L}$ & $\mathrm{~L}$ & 0.29 & $\mathrm{~L}$ \\
\hline desipramine & 3.94 & $\mathrm{H}$ & $\mathrm{H}$ & $\mathrm{H}$ & 4.5 & $\mathrm{H}$ \\
\hline D-glucose & -3.54 & $\mathrm{~L}$ & $\mathrm{H}$ & $\mathrm{H}$ & 10 & $\mathrm{H}$ \\
\hline enalapril & 1.77 & $\mathrm{H}$ & $\mathrm{H}$ & $\mathrm{H}$ & 1.57 & $\mathrm{H}$ \\
\hline enalaprilat & 1.17 & $\mathrm{~L}$ & $\mathrm{~L}$ & $\mathrm{~L}$ & 0.2 & $\mathrm{~L}$ \\
\hline fluvastatin & 3.41 & $\mathrm{H}$ & $\mathrm{H}$ & $\mathrm{H}$ & 2.4 & $\mathrm{H}$ \\
\hline furosemide & 0.74 & $\mathrm{~L}$ & $\mathrm{~L}$ & $\mathrm{~L}$ & 0.05 & $\mathrm{~L}$ \\
\hline hydrochlorothiazide & -0.15 & $\mathrm{~L}$ & $\mathrm{~L}$ & $\mathrm{~L}$ & 0.04 & $\mathrm{~L}$ \\
\hline ketoprofen & 3.31 & $\mathrm{H}$ & $\mathrm{H}$ & $\mathrm{H}$ & 8.7 & $\mathrm{H}$ \\
\hline L-dopa & -2.39 & $\mathrm{~L}$ & $\mathrm{H}$ & $\mathrm{H}$ & 3.4 & $\mathrm{H}$ \\
\hline Lisinopril & 0.91 & $\mathrm{~L}$ & $\mathrm{~L}$ & $\mathrm{~L}$ & 0.33 & $\mathrm{~L}$ \\
\hline L-leucine & -1.62 & $\mathrm{~L}$ & $\mathrm{H}$ & $\mathrm{H}$ & 6.2 & $\mathrm{H}$ \\
\hline Losartan & 4.11 & $\mathrm{H}$ & $\mathrm{H}$ & $\mathrm{L}$ & 1.15 & $\mathrm{~L}$ \\
\hline metoprolol & 1.72 & $\mathrm{R}$ & $\mathrm{R}$ & $\mathrm{R}$ & 1.34 & $\mathrm{R}$ \\
\hline naproxen & 2.86 & $\mathrm{H}$ & $\mathrm{H}$ & $\mathrm{H}$ & 8.5 & $\mathrm{H}$ \\
\hline phenylalanine & 0.07 & $\mathrm{~L}$ & $\mathrm{H}$ & $\mathrm{H}$ & 4.08 & $\mathrm{H}$ \\
\hline piroxicam & 0.29 & $\mathrm{~L}$ & $\mathrm{H}$ & $\mathrm{H}$ & 6.65 & $\mathrm{H}$ \\
\hline propranolol & 2.65 & $\mathrm{H}$ & $\mathrm{H}$ & $\mathrm{H}$ & 2.91 & $\mathrm{H}$ \\
\hline Ranitidine & 0.63 & $\mathrm{~L}$ & $\mathrm{~L}$ & $\mathrm{~L}$ & 0.27 & $\mathrm{~L}$ \\
\hline terbutaline & 1.16 & $\mathrm{~L}$ & $\mathrm{~L}$ & $\mathrm{~L}$ & 0.3 & $\mathrm{~L}$ \\
\hline valacyclovir & -1.06 & $\mathrm{~L}$ & $\mathrm{H}$ & $\mathrm{H}$ & 1.66 & $\mathrm{H}$ \\
\hline verapamil & 5.69 & $\mathrm{H}$ & $\mathrm{H}$ & $\mathrm{H}$ & 6.8 & $\mathrm{H}$ \\
\hline \%Error & & 27.59 & 6.90 & 3.45 & -- & -- \\
\hline
\end{tabular}

${ }^{*}$ Data information obtained from references $1-4$ or drug monographs.

\section{Conclusion}

It is suggested to use more than $90 \%$ metabolism as cut off value to predict high permeability when trying to classify drug permeability classification 


\section{Compliance with Ethical Standards}

Nasir Idkaidek has no conflicts of interest to declare. No funding was received in the preparation of this manuscript.

\section{References}

1. Amidon GL, Lennernäs H, Shah VP, Crison JR (1995) A theoretical basis for a bio pharmaceutic drug classification: the correlation of in vitro drug product dissolution and in vivo bioavailability. Pharm Res 12(3): 413-420.

2. Leslie ZB, Fabio B, Tudor IO (2011) BDDCS Applied to Over 900 Drugs. The AAPS Journal 13(4): 519-547.



3. Benet LZ, Amidon GL, Barends DM, Lennernäs H, Polli JE, et al. (2008) The Use of BDDCS in Classifying the Permeability of Marketed Drugs1. Pharm Res 25(3): 483-488.

4. Kasim NA, Whitehouse M, Ramachandran C, Bermejo M, Lennernäs $\mathrm{H}$, et al. (2004) Molecular Properties of WHO Essential Drugs and Provisional Biopharmaceutical Classification. Mol Pharm 1(1): 85-96.

5. (2015) Guidance for Industry: Waiver of In Vivo Bioavailability and Bioequivalence Studies for Immediate-Release Solid Oral Dosage Forms Based on a Biopharmaceutics Classification System. U.S. Department of Health and Human Services Food and Drug Administration Center for Drug Evaluation and Research (CDER). 5600 Fishers Lane Rockville, MD 20857, USA.

Your next submission with Juniper Publishers
will reach you the below assets
- Quality Editorial service
- Swift Peer Review
- Reprints availability
- E-prints Service
- Manuscript Podcast for convenient understanding
- Global attainment for your research
- Manuscript accessibility in different formats
( Pdf, E-pub, Full Text, Audio)
- Unceasing customer service
Track the below URL for one-step submission
https://juniperpublishers.com/online-submission.php

